\title{
The serum vitamin D level is inversely correlated with nonalcoholic fatty liver disease
}

\author{
Goh Eun Chung ${ }^{1 *}$, Donghee Kim ${ }^{1,2^{*}}$, Min-Sun Kwak', Jong In Yang', Jeong Yoon Yim1', Seon Hee Lim', and Mustafa Itani ${ }^{3}$ \\ 'Department of Internal Medicine, Healthcare Research Institute, Gangnam Healthcare Center, Seoul National University Hospital, \\ Seoul, Korea; ${ }^{2}$ Division of Gastroenterology and Hepatology, Stanford University School of Medicine, Stanford, California, USA; ${ }^{3}$ Pul- \\ monary and Critical Care Consultant, Sheikh Khalifa Specialty Hospital, Ras AlKhaimah, UAE
}

Background/Aims: A low vitamin D level has been associated with metabolic syndrome and diabetes. However, an association between a low vitamin D level and nonalcoholic fatty liver disease (NAFLD) has not yet been definitively established. This study aimed to characterize the relationship between a vitamin D level and NAFLD in Korea.

Methods: A cross-sectional study involving 6,055 health check-up subjects was conducted. NAFLD was diagnosed on the basis of typical ultrasonographic findings and a history of alcohol consumption.

Results: The subjects were aged $51.7 \pm 10.3$ years (mean \pm SD) and $54.7 \%$ were female. NAFLD showed a significant inverse correlation with the vitamin $\mathrm{D}$ level after adjusting for age and sex [odds ratio $(\mathrm{OR})=0.85,95 \%$ confidence interval $(\mathrm{Cl})=0.75-0.96]$. The age- and sex-adjusted prevalence of NAFLD decreased steadily with increasing vitamin $\mathrm{D}$ level [OR=0.74, 95\% Cl=0.60-0.90, lowest quintile $(\leq 14.4 \mathrm{ng} / \mathrm{mL})$ vs highest quintile $(\geq 28.9 \mathrm{ng} / \mathrm{mL}), p$ for trend $<0.001]$. Multivariate regression analysis after adjusting for other metabolic factors revealed that NAFLD showed a significant inverse correlation with both the vitamin D level $(>20 \mathrm{ng} / \mathrm{mL})[\mathrm{OR}=0.86,95 \% \mathrm{Cl}=0.75-0.99]$ and the quintiles of the vitamin $\mathrm{D}$ level in a dose-dependent manner ( $p$ for trend $=0.001$ ).

Conclusions: The serum level of vitamin $D$, even when within the normal range, was found to be inversely correlated with NAFLD in a dose-dependent manner. Vitamin D was found to be inversely correlated with NAFLD independent of known metabolic risk factors. These findings suggest that vitamin D exerts protective effects against NAFLD.

(Clin Mol Hepatol 2016;22:146-151)

Keywords: Fatty liver; Vitamin D; Metabolic factor

\section{INTRODUCTION}

Nonalcoholic fatty liver disease (NAFLD) is the leading cause of chronic liver disease with an approximate prevalence of 20-30\% in the Western countries and $16-33 \%$ in Korea. ${ }^{1}$ As the pathogenesis of NAFLD is closely related to insulin resistance, ${ }^{2,3}$ the devel- opment of NAFLD has been related to various components of metabolic syndrome such as, dyslipidemia, obesity, type II diabetes and cardiovascular disease. ${ }^{4}$ Therefore, NAFLD has been considered to be a hepatic manifestation of metabolic syndrome. ${ }^{5}$

Vitamin $D$ is a fat-soluble vitamin, that affects metabolism including immune function and bone metabolism. ${ }^{6}$ It is formed in

\section{Abbreviations:}

ALT, alanine aminotransferase; AST, aspartate aminotransferase; BMI, body mass index; GGT, gamma-glutamyltransferase; $\mathrm{HDL}$, high-density lipoprotein; LDL, low-density lipoprotein; NAFLD, nonalcoholic fatty liver disease

\section{Corresponding author: Goh Eun Chung}

Department of Internal Medicine, Healthcare Research Institute, Gangnam Healthcare Center, Seoul National University Hospital, 152 Teheran-ro, Gangnam-gu, Seoul 06236, Korea

Tel: +82-2-2112-5741, Fax: +82-2-2112-5635

E-mail: gohwom@hanmail.net 
the skin or obtained from the diet. In the liver, vitamin D can be metabolized to 25-hydroxyvitamin [25(OH)D], which is its major circulating metabolite. Many studies have linked low levels of vitamin with metabolic syndrome, type II diabetes mellitus (DM), metabolic bone diseases and obesity. ${ }^{7-9}$ A recent meta-analysis of both Western and Eastern cohort studies showed that NAFLD patients have decreased serum 25(OH)D concentrations. ${ }^{10}$ However, few studies of vitamin D levels and NAFLD in populations in Korea have been previously reported. In Korea, a cross-sectional study showed that lower $25(\mathrm{OH}) \mathrm{D}_{3}$ levels were associated with a significantly increased risk of NAFLD, independent of obesity and metabolic syndrome. ${ }^{11}$ However, in the previous study, the cohort included only males. Therefore, we aimed herein to investigate the association between NAFLD and serum vitamin D levels in the apparently healthy individuals in Korea.

\section{PATIENTS AND METHODS}

\section{Study population}

A cross-sectional study was performed to investigate the association between the serum concentrations of vitamin $D$ and NAFLD. Participants who examined serum vitamin D tests and abdominal ultrasonography at the Seoul National University Hospital Gangnam Healthcare Center in Seoul, Republic of Korea for routine health check-ups from January 2010 to September 2010 were recruited. Most of the subjects spontaneously paid for their health check-ups, while some of the participants were supported by their employer.

We excluded subjects with other potential causes of chronic liver disease, including 291 subjects positive for hepatitis B virus, 134 for positive for hepatitis C virus, and 598 with a history of significant alcohol consumption (>30 g/day for males and >20 g/ day for females) or a history of hepatitis from other causes. Ultimately, 5,409 subjects were enrolled. This study was approved by the Institutional Review Board of the Seoul National University Hospital with a waiver of informed consent.

\section{Clinical and laboratory assessments}

Each subject completed a questionnaire regarding their past medical history and lifestyle. All subjects participated in anthropometric assessment and the laboratory and radiologic tests on the same day. Height and body weight were measured using a digital scale, and body mass index (BMI) was calculated by dividing the weight $(\mathrm{kg})$ by the square of the height $\left(\mathrm{m}^{2}\right)$. Waist circumference was measured at the midpoint between the lower costal margin and the anterior superior iliac crest by a well-trained examiner using a tape. Blood pressures were measured twice, and the mean values were reported. Hypertension was defined as either a systolic blood pressure $\geq 140 \mathrm{mmHg}$, a diastolic blood pressure $\geq 90 \mathrm{mmHg}$ or current use of anti-hypertensive medication. Serum samples were collected after 12 hours overnight fast. DM was defined as a fasting glucose level $\geq 126 \mathrm{mg} / \mathrm{dL}$ or current use of anti-diabetic medication. Laboratory tests included serum alanine aminotransferase (ALT), aspartate aminotransferase (AST), gamma-glutamyl transferase (GGT), fasting glucose, triglycerides, total cholesterol, low-density lipoprotein (LDL) cholesterol, highdensity lipoprotein (HDL) cholesterol, hepatitis B surface antigen and antibody to hepatitis $C$ virus.

\section{Serum vitamin D levels}

Serum levels of 25-hydroxyvitamin $(\mathrm{OH}) \mathrm{D}_{3}$ were measured using a chemiluminescnece immunoassay kit (Diasorin, Stillwater OK, OK ,USA). Subjects were categorized as having either low vitamin D levels $(<20 \mathrm{ng} / \mathrm{mL})$, or normal vitamin D levels $(\geq 20 \mathrm{ng} / \mathrm{mL}){ }^{12}$ Additionally, quintile 1 of serum vitamin D level was $\leq 14.4 \mathrm{ng} / \mathrm{mL}$, quintile 2 was $14.5-18.8 \mathrm{ng} / \mathrm{mL}$, quintile 3 was $18.9-23.1 \mathrm{ng} / \mathrm{mL}$, quintile 4 was $23.2-28.8 \mathrm{ng} / \mathrm{mL}$, and quintile 5 was $\geq 28.9 \mathrm{ng} / \mathrm{mL}$. We used the lowest quintile $(\leq 14.4 \mathrm{ng} / \mathrm{mL})$ of vitamin $D$ levels as a reference group.

\section{Assessment of NAFLD}

NAFLD was defined as the presence of fatty liver as determined via ultrasonography in the absence of the following: 1) hepatitis $B$ surface antigen seropositivity or antibody to hepatitis C virus, 2) significantly excessive alcohol consumption (>30 g/day for males or $>20 \mathrm{~g} /$ day for females), 3) medications previously known to induce fatty liver disease, and 4) other known causes of hepatic disease. Ultrasonographic examinations of the liver were performed by experienced radiologists who were blinded to the clinical information using a 3.5-MHz transducer (Acusion, Sequoia 512, Siemens, Mountain View, CA, USA). Diagnoses of fatty liver were based on the following previously established standardized definitions $^{13}$ : hepatorenal echo contrast, liver brightness, deep attenuation and vascular blurring. 
Table 1. Comparison of baseline characteristics between subjects with and without nonalcoholic fatty liver disease

\begin{tabular}{|c|c|c|c|}
\hline & $\begin{array}{c}\text { NAFLD } \\
(n=1,660)\end{array}$ & $\begin{array}{c}\text { Control } \\
(n=3,749)\end{array}$ & $P$-value \\
\hline Age (years) & $53.6 \pm 9.5$ & $50.9 \pm 10.5$ & $<0.001$ \\
\hline Male (\%) & $1,069(64.4)$ & $1,382(36.9)$ & $<0.001$ \\
\hline Diabetes mellitus (\%) & $121(7.3)$ & $103(2.70)$ & $<0.001$ \\
\hline Body mass index $\left(\mathrm{kg} / \mathrm{m}^{2}\right)$ & $25.29 \pm 2.92$ & $22.30 \pm 2.67$ & $<0.001$ \\
\hline Waist circumference (cm) & $89.63 \pm 8.03$ & $81.10 \pm 7.99$ & $<0.001$ \\
\hline AST (IU/L) & $25.9 \pm 12.4$ & $21.2 \pm 7.5$ & $<0.001$ \\
\hline $\mathrm{ALT}(\mathrm{IU} / \mathrm{L})$ & $31.6 \pm 20.6$ & $19.7 \pm 11.3$ & $<0.001$ \\
\hline Cholesterol (mg/dL) & $198.9 \pm 36.0$ & $193.1 \pm 33.6$ & $<0.001$ \\
\hline Triglycerides (mg/dL) & $144.8 \pm 84.0$ & $88.7 \pm 50.0$ & $<0.001$ \\
\hline HDL cholesterol (mg/dL) & $48.0 \pm 10.0$ & $55.4 \pm 12.0$ & $<0.001$ \\
\hline Fasting glucose (mg/dL) & $104.8 \pm 21.8$ & $93.4 \pm 13.5$ & $<0.001$ \\
\hline $25(\mathrm{OH})$ Vitamin $\mathrm{D}_{3}(\mathrm{ng} / \mathrm{mL})$ & $21.75 \pm 7.54$ & $22.02 \pm 8.43$ & 0.253 \\
\hline
\end{tabular}

Data represent means $\pm S D$.

AST, aspartate aminotransferase; ALT, alanine aminotransferase; GGT, gamma-glutamyltransferase; HDL, high-density lipoprotein; NAFLD, nonalcoholic fatty liver disease

\section{Statistical analysis}

Categorical variables were compared using a chi-square test or Fisher's exact test and continuous variables between the two groups were performed using the Student's $t$-test. Logistic regression analysis was used to analyze the associations between NAFLD and serum levels of $25(\mathrm{OH}) \mathrm{D}_{3}$. Multivariate logistic regression analysis was performed to identify independent risk factors after adjusting other confounders. Statistical analyses were performed using SPSS 19.0 (SPSS Inc.; Chicago, IL, USA). P-value $<0.05$ was considered statistically significant.

\section{RESULTS}

\section{Study population}

A total of 5,409 subjects (mean age $51.7 \pm 10.3$, female $54.7 \%$ ) were analyzed. Of the 5,409 subjects, 1,660 (30.7\%) subjects had ultrasonographically diagnosed NAFLD. Table 1 compares the subjects with and without NAFLD. Individuals with NAFLD showed an older age, a higher prevalence of DM and hypertension, higher BMls, waist circumference, AST, ALT, GGT, triglycerides, HDL-cholesterol and fasting glucose and were also more fre- quently of the male gender compared with those without NAFLD. There was no significant difference in the levels of $25(\mathrm{OH}) \mathrm{D}_{3}$ between the two groups.

\section{Relationship between $25(\mathrm{OH}) \mathrm{D}_{3}$ and NAFLD}

To analyze the relationship between NAFLD and the levels of $25(\mathrm{OH}) \mathrm{D}_{3}$, multivariable binary and ordinal analyses were performed with NAFLD as a dependent variable (Table 2). NAFLD was significantly inversely associated with vitamin $D$ levels after adjusting for age and sex [odds ratio (OR) $0.85,95 \%$ confidence interval (CI) $0.75-0.96]$. The age and sex-adjusted prevalence of NAFLD decreased steadily with increasing levels of vitamin D [OR $0.74,95 \% \mathrm{Cl}=0.60-0.90$, lowest quintile $(\leq 14.4 \mathrm{ng} / \mathrm{mL})$ vs highest quintile $(\geq 28.9 \mathrm{ng} / \mathrm{mL}), p$ for trend $<0.001]$. Multivariate regression analysis after adjusting for BMl, waist circumference, triglycerides, HDL cholesterol, LDL cholesterol, diabetes, and hypertension revealed that NAFLD was statistically significantly inversely associated with vitamin D levels ( $>20 \mathrm{ng} / \mathrm{mL}$ ) [OR 0.87 , $95 \% \mathrm{Cl}, 0.75-0.99]$ and the grade of vitamin D levels in a dosedependent manner [OR=0.93, 95\% Cl 0.74-1.16 in 3rd quintile $(18.9-23.1 \mathrm{ng} / \mathrm{mL})$, OR $0.89,95 \% \mathrm{Cl} 0.71-1.11$ in the 4th quintile (23.2-28.8 $\mathrm{ng} / \mathrm{mL}$ ) and $\mathrm{OR} 0.73,95 \% \mathrm{Cl}, 0.58-0.91$ in 5th quintile vs the lowest quintile, $p$ for trend $=0.002]$. 
Table 2. Age- and sex-adjusted and multivariable binary and ordinal analyses of the risk of nonalcoholic fatty liver disease

\begin{tabular}{|c|c|c|c|c|c|c|}
\hline \multirow[t]{2}{*}{ Vitamin D3 } & $\begin{array}{c}\text { Age, } \\
\text { sex adjusted }\end{array}$ & \multirow[t]{2}{*}{$P$-value } & $\begin{array}{c}\text { Multivariable } \\
\text { model } 1\end{array}$ & \multirow[t]{2}{*}{$P$-value } & $\begin{array}{l}\text { Multivariable } \\
\text { model } 2\end{array}$ & \multirow[t]{2}{*}{$P$-value } \\
\hline & OR $(95 \% \mathrm{Cl})$ & & OR $(95 \% \mathrm{Cl})$ & & OR $(95 \% \mathrm{Cl})$ & \\
\hline$\leq 20 \mathrm{ng} / \mathrm{mL}$ & 1 (reference) & & 1 (reference) & & 1 (reference) & \\
\hline$>20 \mathrm{ng} / \mathrm{mL}$ & $0.85(0.75-0.96)$ & 0.010 & $0.86(0.75-0.99)$ & 0.032 & $0.87(0.75-0.99)$ & 0.049 \\
\hline \multicolumn{7}{|l|}{ Quintile } \\
\hline 1st (-14.4) & 1 (reference) & $0.001^{*}$ & 1 (reference) & $0.003^{*}$ & 1 (reference) & $0.002^{*}$ \\
\hline 2nd (14.5-18.8) & 1.14 (0.94-1.38) & & $1.09(0.88-1.35)$ & & $1.07(0.85-1.34)$ & \\
\hline $3 r d(18.9-23.1)$ & $0.96(0.79-1.17)$ & & $0.93(0.76-1.15)$ & & $0.93(0.74-1.16)$ & \\
\hline 4th (23.2-28.8) & $0.95(0.79-1.16)$ & & $0.93(0.75-1.15)$ & & $0.89(0.71-1.11)$ & \\
\hline 5th (28.9-) & $0.74(0.61-0.90)$ & & $0.75(0.60-0.93)$ & & $0.73(0.58-0.91)$ & \\
\hline
\end{tabular}

Multivariable model 1 was adjusted for age, sex, body mass index, waist circumference, diabetes, and hypertension. Multivariable model 2 includes diabetes, hypertension, low-density lipoprotein cholesterol, and high-density lipoprotein cholesterol in addition to the variables addressed in model 1.

$\mathrm{OR}$, odds ratio; $\mathrm{Cl}$, confidence interval.

${ }^{*} P$-value for test of trend of odds.

\section{DISCUSSION}

In the present study, we found that serum concentrations of vitamin $D$, even within the normal range, were inversely associated with NAFLD in a dose-dependent manner. This association was independent of various well-identified metabolic risk factors for NAFLD, including age, sex, BMI, waist circumference, DM, hypertension, and levels of LDL- and HDL-cholesterol. These findings suggest an important role for vitamin $D$ in the pathogenesis of NAFLD.

In accord with our findings, many previous studies have suggested inverse relationships between vitamin D and NAFLD. A systematic review showed that NAFLD patients were 1.26-times more likely to be vitamin $D$ deficient. Interestingly, these differences were higher in Western populations than in Eastern populations. ${ }^{10}$ Targher et al..$^{14}$ reported that patients with biopsy-confirmed NAFLD showed a marked reduction in serum $25(\mathrm{OH}) \mathrm{D}_{3}$ levels compared with controls. In another study of 262 subjects performed in Italy, low $25(\mathrm{OH}) \mathrm{D}_{3}$ levels were associated with NAFLD independent of insulin-resistance, diabetes and metabolic syndrome..$^{15}$ In contrast, a Chinese population based-survey showed that vitamin D status was not significantly associated with NAFLD. ${ }^{16}$

However, few studies have assessed the association between vitamin D levels and NAFLD in Korea. In a recent study of healthy Korean men, the proportion of NAFLD patients increased as the serum $25(\mathrm{OH}) \mathrm{D}_{3}$ levels decreased and multiple logistic regression analyses showed that the lower $25(\mathrm{OH}) \mathrm{D}_{3}$ tertile levels were asso- ciated with a significantly increased risk of NAFLD compared with the highest tertile after adjusting for metabolic syndrome. ${ }^{10}$ However, that study included only men, which introduced a selection bias. Another study performed with a population-based cohort in Korea reported that low vitamin D status was closely associated with NAFLD, independent of visceral fat, as measured by computed tomography. ${ }^{17}$ The results of the present study, which had a relatively large-scale cohort that included both men and women confirmed these findings. In our study, although the highest quintile of vitamin D showed significantly inverse relationship with the presence of NAFLD, there was no significant risk increase of NAFLD according to low vitamin D levels from 2nd to 4th quintiles. These results suggest the protective effect of vitamin $D$ on NAFLD is more profound when serum levels of vitamin D level are more sufficient.

The underlying mechanism for the role of vitamin $D$ in the development of NAFLD has not been clearly elucidated. In a previous animal study, rats fed with vitamin D depletion, the vitamindeficient group showed greater hepatic steatosis and inflammation through toll-like receptor activation compared with the control group..$^{18}$

Median vitamin D concentrations varied among previous studies. However, the reasons for these differences are unclear. Ultraviolet $B$ exposure, dietary vitamin $D$ consumption, vitamin $D$ fortification habits in various countries and differences in the season of samplings may have resulted in the different findings. ${ }^{19}$ Moreover, the $25(\mathrm{OH}) \mathrm{D}$ assays were not standardized because of the use of different assay materials. ${ }^{20,21}$ To date, there is no consensus 
on ideal cut-off levels for vitamin D deficiency, and various factors such as geography, sex and season may need to be considered.

There were several drawbacks in this study. First, its cross-sectional design made it difficult to confirm temporal associations between vitamin D levels and NAFLD. Second, we could not obtain results of liver histology, which is the gold standard for NAFLD diagnosis. Although, ultrasonography might introduce false-negative outcomes when fatty infiltration of the liver falls below $30 \%$, it is not possible to perform invasive tests in evidently healthy population. Therefore, ultrasonography was used as a first-line method to diagnose NAFLD according to current clinical guidelines. ${ }^{23,24}$ Third, we could not exclude subjects who were taking vitamin D supplements and we did not consider seasonal variations of vitamin $D$ levels in our analysis. Finally, we could not obtain data of physical activity such as exercise or outdoor activity, which could be a confounding factor for both NAFLD and serum vitamin D levels.

In conclusion, our findings show that serum vitamin $D$, even within the normal range, was found to be inversely associated with NAFLD in a dose-dependent manner. Vitamin D levels were inversely associated with NAFLD independent of known metabolic risk factors. Thus, vitamin D may exert a protective effect against NAFLD.

\section{Acknowledgments}

This study was supported by grant no. 34-2014-0240 from the SK Telecom Research Fund.

\section{Conflicts of Interest}

The authors have no conflicts to disclose.

\section{REFERENCES}

1. Vernon G, Baranova A, Younossi ZM. Systematic review: the epidemiology and natural history of non-alcoholic fatty liver disease and non-alcoholic steatohepatitis in adults. Aliment Pharmacol Ther 2011;34:274-285.

2. Almeda-Valdés P, Cuevas-Ramos D, Aguilar-Salinas CA. Metabolic syndrome and non-alcoholic fatty liver disease. Ann Hepatol 2009;8(Suppl 1):S18-S24.

3. Khashab MA, Liangpunsakul S, Chalasani N. Nonalcoholic fatty liver disease as a component of the metabolic syndrome. Curr Gastroenterol Rep 2008;10:73-80.

4. Kim D, Choi SY, Park EH, Lee W, Kang JH, Kim W, et al. Nonalcoholic fatty liver disease is associated with coronary artery calcification.
Hepatology 2012;56:605-613.

5. Chen SH, He F, Zhou HL, Wu HR, Xia C, Li YM. Relationship between nonalcoholic fatty liver disease and metabolic syndrome. J Dig Dis 2011;12:125-130.

6. Hewison M. An update on vitamin D and human immunity. Clin Endocrinol (Oxf) 2012;76:315-325.

7. Gagnon C, Lu ZX, Magliano DJ, Dunstan DW, Shaw JE, Zimmet PZ, et al. Low serum 25-hydroxyvitamin $D$ is associated with increased risk of the development of the metabolic syndrome at five years: results from a national, population-based prospective study (The Australian Diabetes, Obesity and Lifestyle Study: AusDiab). J Clin Endocrinol Metab 2012;97:1953-1961.

8. Kim MK, Baek KH, Song KH, II Kang M, Park CY, Lee WY, et al. Vitamin D deficiency is associated with sarcopenia in older Koreans, regardless of obesity: the Fourth Korea National Health and Nutrition Examination Surveys (KNHANES IV) 2009. J Clin Endocrinol Metab 2011;96:3250-3256.

9. Lim S, Shin H, Kim MJ, Ahn HY, Kang SM, Yoon JW, et al. Vitamin D inadequacy is associated with significant coronary artery stenosis in a community-based elderly cohort: the Korean Longitudinal Study on Health and Aging. J Clin Endocrinol Metab 2012;97:169-178.

10. Eliades M, Spyrou E, Agrawal N, Lazo M, Brancati FL, Potter JJ, et al. Meta-analysis: vitamin D and non-alcoholic fatty liver disease. Aliment Pharmacol Ther 2013:38:246-254.

11. Rhee EJ, Kim MK, Park SE, Park CY, Baek KH, Lee WY, et al. High serum vitamin $D$ levels reduce the risk for nonalcoholic fatty liver disease in healthy men independent of metabolic syndrome. Endocr J 2013;60:743-752.

12. Slomski $A$. IOM endorses vitamin $D$, calcium only for bone health, dispels deficiency claims. JAMA 2011;305:453-454.

13. Saadeh S, Younossi ZM, Remer EM, Gramlich T, Ong JP, Hurley M, et al. The utility of radiological imaging in nonalcoholic fatty liver disease. Gastroenterology 2002;123:745-750.

14. Targher G, Bertolini L, Scala L, Cigolini M, Zenari L, Falezza G, et al. Associations between serum 25-hydroxyvitamin D3 concentrations and liver histology in patients with non-alcoholic fatty liver disease. Nutr Metab Cardiovasc Dis 2007;17:517-524.

15. Barchetta I, Angelico F, Del Ben M, Baroni MG, Pozzilli P, Morini S, et al. Strong association between non alcoholic fatty liver disease (NAFLD) and low 25(OH) vitamin D levels in an adult population with normal serum liver enzymes. BMC Med 2011;9:85.

16. Li L, Zhang L, Pan S, Wu X, Yin X. No significant association between vitamin $D$ and nonalcoholic fatty liver disease in a Chinese population. Dig Dis Sci 2013;58:2376-2382.

17. Seo JA, Eun CR, Cho H, Lee SK, Yoo HJ, Kim SG, et al. Low vitamin $D$ status is associated with nonalcoholic Fatty liver disease independent of visceral obesity in Korean adults. PLoS One 2013;8:e75197.

18. Roth CL, Elfers CT, Figlewicz DP, Melhorn SJ, Morton GJ, Hoofnagle 
A, et al. Vitamin D deficiency in obese rats exacerbates nonalcoholic fatty liver disease and increases hepatic resistin and Toll-like receptor activation. Hepatology 2012;55:1103-1111.

19. Schöttker B, Jorde R, Peasey A, Thorand B, Jansen EH, Groot Ld, et al. Vitamin $D$ and mortality: meta-analysis of individual participant data from a large consortium of cohort studies from Europe and the United States. BMJ 2014;348:g3656.

20. Schöttker B, Jansen EH, Haug U, Schomburg L, Köhrle J, Brenner H. Standardization of misleading immunoassay based 25-hydroxyvitamin D levels with liquid chromatography tandem-mass spectrometry in a large cohort study. PLoS One 2012;7:e48774.

21. Carter GD, Carter R, Jones J, Berry J. How accurate are assays for 25-hydroxyvitamin $D$ ? Data from the international vitamin $D$ exter- nal quality assessment scheme. Clin Chem 2004;50:2195-2197.

22. Sanyal AJ. AGA technical review on nonalcoholic fatty liver disease. Gastroenterology 2002;123:1705-1725.

23. Korean Association for the Study of the Liver (KASL). KASL clinical practice guidelines: management of nonalcoholic fatty liver disease. Clin Mol Hepatol 2013;19:325-348.

24. Chalasani N, Younossi Z, Lavine JE, Diehl AM, Brunt EM, Cusi K, et al. The diagnosis and management of non-alcoholic fatty liver disease: practice guideline by the American Gastroenterological Association, American Association for the Study of Liver Diseases, and American College of Gastroenterology. Gastroenterology 2012;142:1592-1609. 